\title{
Neonatal circumcision in patients with haemophilia is safe - a single centre experience
}

By Assaf Arie Barg, Sarina Levy Mendelovich, Shadan Lalezari, Shirley Azar Avivi, Dalia Bashari, Gili Kenet

Consensus evidence-based guidelines regarding prophylactic treatment for circumcision in patients with haemophilia are lacking. In this study, we retrospectively examined the results of circumcision of 40 consecutive neonates with haemophilia, diagnosed after delivery. Our protocol included application of a single dose of factor replacement prior to the procedure and 3 days' oral treatment with tranexamic acid. Only 3/40 neonates with haemophilia experienced mild postcircumcision bleeding. No major or any life-threatening bleeds were encountered. As the therapy administered was minimal and the procedure simple and feasible, we concluded that circumcision in neonates with haemophilia should be performed according to family's and physician's discretion. Avoidance of circumcising

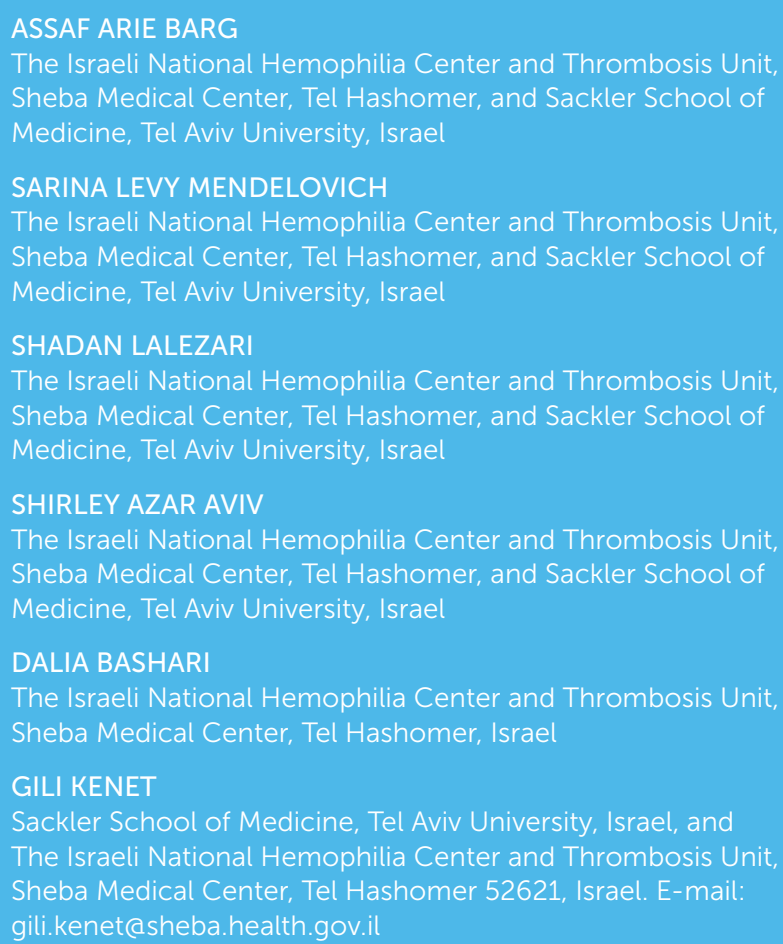

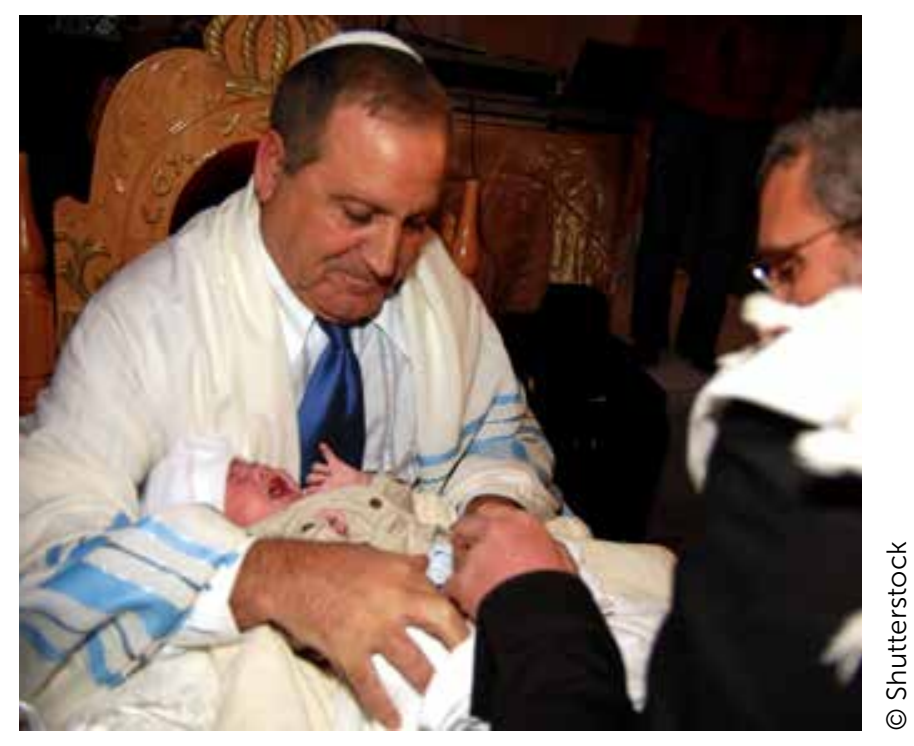

male babies with haemophilia due to fear of bleeding complications is not supported by the results shown within our patient cohort.

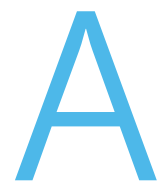

$n$ estimated one in three males worldwide is circumcised ${ }^{[1]}$. In Israel the vast majority of the population is either Jewish or Islamic; ritual circumcision has been practiced by Jews and Muslims for centuries. Due to religious, traditional and cultural reasons, circumcision is the most frequent surgical procedure performed. Currently, most males in Israel are circumcised as neonates, with more than 70 circumcisions performed daily ${ }^{[2]}$. The vast majority of patients with haemophilia in Israel are circumcised as neonates.

Neonatal circumcision in patients with haemophilia has not been well studied. A recent survey among paediatric haematologists in the United States revealed significant variation in approaches to circumcision in boys with haemophilia ${ }^{[3]}$. The majority of those surveyed opposed circumcision in neonates with 
haemophilia due to concerns of potential bleeding, inhibitor formation (that may be associated with early exposure to replacement therapy ${ }^{[4-5]}$ ) and issues related to venous access in neonates. All respondents used factor replacement prior to circumcision and most $(78 \%$ of respondents) used additional postoperative doses of factor.

The aim of the study reported here was to evaluate the efficacy, safety and complication rates of our own institutional protocol for circumcision of neonates with haemophilia.

\section{Methodology}

The Israeli National Hemophilia Institute takes care of over 660 patients with haemophilia. Despite an active national plan for carrier detection and prenatal counselling, 7-10 babies with haemophilia are born in Israel every year. Whereas sporadic cases are diagnosed very early, due to post- circumcision bleed, all other pre-diagnosed patients are advised to be treated using the same institutional protocol and recommendations.

We retrospectively reviewed computerised medical records of all new haemophilia patients registered in the Israeli National Hemophilia Center from January 2010 to July 2016. Demographic data concerning haemophilia type and severity was collected, as well as data regarding post-circumcision bleeding and factor replacement requirements. In addition, we checked for inhibitor formation.

Our institutional protocol for neonatal haemophilia patients undergoing elective circumcision includes one dose of factor replacement at a fixed dose of 250 units (50-80 units per $\mathrm{kg}$ ), regardless of haemophilia type, given 1-6 hours prior to the procedure. Patients are treated for three to five days after the day of circumcision with tranexamic acid at a dose of $10-20 \mathrm{mg}$ per $\mathrm{kg}$, mostly $50 \mathrm{mg}$ a dose, three to four times a day.

\section{Results}

Data for 40 neonates with haemophilia in whom elective circumcision was planned according to our protocol was available. Demographic data regarding haemophilia type and severity is presented in Figure 1. 18 out of 40 patients had severe haemophilia; 29

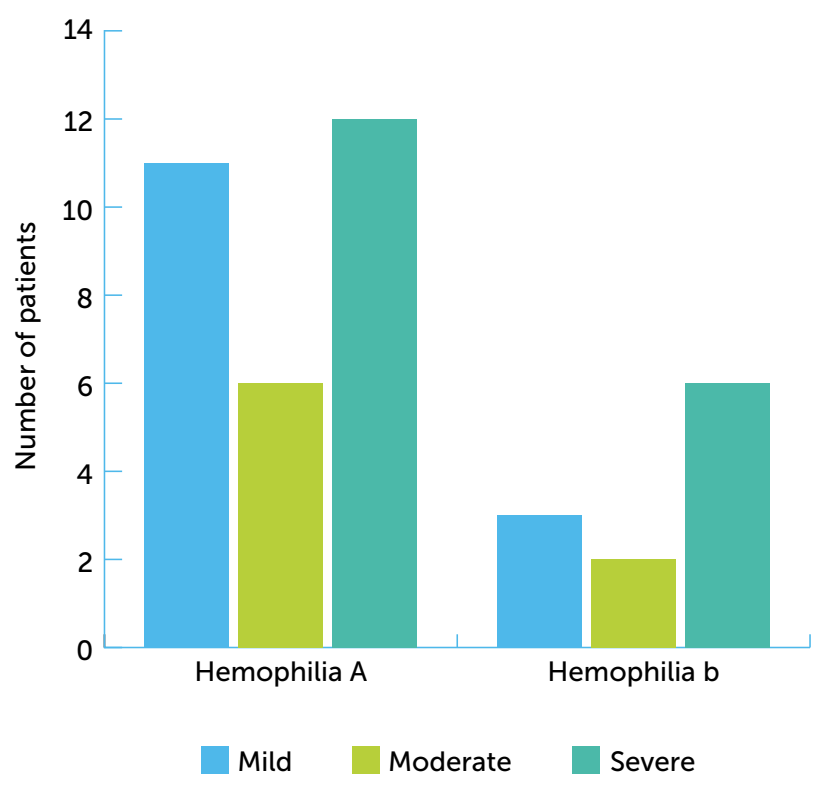

Figure 1: Demographic data regarding haemophilia type and severity for the Tel Hashomer cohort

patients had haemophilia A. Data concerning bleeding rates and inhibitor formation, comparing haemophilia $\mathrm{A}$ and $\mathrm{B}$, is presented (Table 1).

Three patients required additional factor replacement therapy due to post-circumcision bleeding (7.5\%). All three were term infants with severe haemophilia A. Two were diagnosed due to previous family history; one was diagnosed due to excessive bleeding post-perinatal heel prick. One did not comply with the protocol as circumcision was performed 24 hours post-factor infusion. In all three cases, the bleeding was mild and did not result in haemoglobin decrease or require blood transfusion.

There was no known family history of inhibitor in any of the cases. Interestingly, inhibitor formation was later noted in one of the patients who experienced post-circumcision bleeding and in two that did not. None of the patients with haemophilia B experienced post circumcision bleeding.

\section{Discussion}

Bleeding is the most common complication related to circumcision, even for healthy boys, with a reported

Table 1 Circumcision data according to haemophilia type

\begin{tabular}{l|l|l|} 
& HAEMOPHILIA A & HAEMOPHILIA B \\
\hline Median age at circumcision (days) & 8 & 8 \\
\hline Post-circumcision bleeding rate & $3 / 29$ & $0 / 11$ \\
\hline Inhibitor formation & $3 / 29$ & $0 / 11$ \\
\hline
\end{tabular}


incidence of $0.1-35 \%(6)$. Potential bleeding is a dreaded complication in patients with haemophilia and data concerning effective prophylactic treatment is scarce. To the best of our knowledge, this is the first study evaluating the safety of circumcision of haemophilic patients specifically at the neonatal period.

Our protocol was effective in preventing postcircumcision bleeding in the majority of neonates with haemophilia. Bleeding rates in our group were similar to protocols using additional post-circumcision prophylactic doses, such as the Izmir protocol used in Turkey ${ }^{[7]}$.

Despite the reluctance of many clinicians to perform circumcision in haemophilic patients during the neonatal period, the current study demonstrates the relative safety of performing the procedure in this young age group. The fact that none of the patients with haemophilia $B$ in our study experienced post-circumcision bleeding may be attributed to the potentially milder expression of this disease as compared to haemophilia A, as has been suggested previously ${ }^{[8-9]}$.

The rate of inhibitors detected later, during followup of our small cohort ( 0 in patients with haemophilia B, about $30 \%$ in patients with severe haemophilia A), was similar to the literature reports ${ }^{[10]}$. As it was not affected by early factor administration or related to post-circumcision mild bleed, circumcision should not be considered as a potential risk factor for inhibitor formation and carries no future risk.

It is important to note that this study's results apply only to neonates. Studies concerning haemostasis in the neonatal period indicate the presence of higher von Willebrand levels and multimers, which may yield some protective impact when post-procedural bleeding occurs ${ }^{[11]}$. Circumcision in older patients may require additional factor doses. Furthermore, a major advantage of neonatal circumcision is that suturing is usually unnecessary, whereas it is commonly used in circumcisions in older age groups ${ }^{[1]}$.

Limitations of this study include retrospective data collection and the small size of the group. Further studies are required in order to elucidate the safest protocol for neonates with haemophilia undergoing circumcision, while avoiding unnecessary exposure to factor replacement therapy at this early age.

\section{Conclusion}

Neonatal circumcision in haemophilia is common and our study suggests that it may be performed safely using single factor replacement therapy and tranexamic acid. Further prospective studies should be performed in order to devise an evidenced-based protocol.

\section{Disclosures}

The authors have advised no interests that might be perceived as posing a conflict or bias.

This is an Open Access article distributed under the terms of the Creative Commons Attribution License (http://creativecommons.org/licenses/by/2.0), which permits unrestricted use, distribution, and reproduction in any medium, provided the original work is properly cited.

\section{References}

1. Weiss HA, Larke N, Halperin D, Schenker I. Complications of circumcision in male neonates, infants and children: a systematic review. BMC Urology; 2010: 10:2. doi:10.1186/1471-2490-10-2.

2. Niku SD, Stock JA, Kaplan GW. Neonatal circumcision. Urol Clin N Am 1995; 22: 5765

3. Kearney S, Sharathkumar A, Rodriguez $V$, et al. Neonatal circumcision in severe haemophilia: a survey of paediatric haematologists at United States Hemophilia Treatment Centers. Haemophilia 2015; 21: 52-7.

4. Gouw SC, van der Bom JG, van den Berg HM. Treatmentrelated risk factors of inhibitor development in previously untreated patients with hemophilia A: the CANAL cohort study. Blood 2007; 109: 4648-54.

5. Gouw SC, van den Berg HM, Fischer K, et al. Intensity of factor VIII treatment and inhibitor development in children with severe hemophilia A: the RODIN study. Blood 2013; 121(20): 4046-55.

6. Kavalki K, Aledort LM. Circumcision and haemophilia: a perspective. Haemophilia 1998; 4: 1-3.

7. Ylilmaz $D, A$ kin $M, A y$, et al. A single centre experience in circumcision of haemophilia patients: Izmir protocol. Haemophilia 2010; 16: 888-91.

8. Makris M. Is VIII worse than IX? Blood 2009; 114(4): 750-1.

9. Melchiorre D, Linari S, Manetti $M$, et al. Clinical, instrumental, serological and histological findings suggest that hemophilia B may be less severe than hemophilia A. Haematologica. 2016 101(2): 219-25.

10. Eckhardt CL, van Velzen AS, Peters M, et al. Factor VIII gene (F8) mutation and risk of inhibitor development in non-severe hemophilia A. Blood 2013; 122(11): 1954-62.

11. Andrew M. Developmental hemostasis: relevance to hemostatic problems during childhood. Semin Thromb Hemost 1995; 21: 341-56. 